\section{PTH-086 REGIONAL IBD SURVEILLANCE ENDOSCOPY NORTH- WEST (RISE NOW): A PROSPECTIVE AUDIT OF PRACTICE IN NORTH-WEST ENGLAND}

Gastroenterology Trainee Research and Improvement Network North West GasTRIN NoW* Pennine Acute NHS Hospitals Trust, Greater Manchester, UK

\subsection{6/gutjnl-2019-BSGAbstracts.145}

Introduction Interval surveillance colonoscopy plays a crucial role in identifying and managing colitis related dysplasia to reduce the risk of colorectal cancer. Dye based or image enhanced chromoendoscopy has been endorsed by multiple organisations as the preferred means of detecting dysplasia since 2015. We aimed to assess the methods of surveillance utilised within the North West of England using the established trainee research network, Gastroenterology Trainee Research and Improvement Network North West (GasTRIN NoW).

Methods GasTRIN NoW investigators prospectively collected data from 10 hospitals in North West England to assess surveillance practice between June and October 2018. All IBD interval surveillance colonoscopies were included. SCENIC consensus guidelines were used as the standard for adequate surveillance while BSG standards were used for the interval surveillance standard. ${ }^{12}$

Results 226 patients underwent IBD surveillance endoscopy (143 UC, 66 CD, 17 IBDU) with a median disease furation of 12 years (IQR9-20). There were 122 males and the median age was 54 years (range 20-86). A total of 46 (20\%) procedures did not adhere to guidelines and 21 (46\%) were delayed ( $>6$ months). Dye spray was used in $22 \%(n=49)$ of procedures while the remaining had random colonic biopsies. Image enhanced chromoendoscopy was not used in our cohort. There was more visible dysplasia identified in the dye spray cohort 913 dye spray vs. 8 non dye spray, chi sq $\mathrm{p}=7 \times\left(0^{-6}\right)$. Adenocarcinoma was confirmed in the dye spray groupo while no cancers were identified in the non dye spray group. There were no differences in histoligical dysplasia between these groups (5 vs. 6 respectively, $\mathrm{p}=0.11$ ). Where withdrawal time was recorded $(n=139)$, median times were significantly different between both groups (dye spray $16 \mathrm{~min}$ (IQR 12-25) vs. no dye spray $10 \mathrm{~min}$ (8-14 min); Wilcox test, $\mathrm{p}=3.7 \times 10^{-4}$ ).

Conclusions Our data demonstrates that there are delays to elective IBD surveillance in clinical practice. Dye spray colonoscopy is not widely practised across North west England. Dye spray colonoscopy identified more visible dysplasia and was associated with longer withdrawal time, a recognised surrogate marker for colonoscopy quality. Our data will inform future work in optimising IBD surveillance in England.

\section{REFERENCES}

1. Laine L, Kaltenbach T, Barkun A, et al. SCENIC international consensus statement on surveillance and management of dysplasia in inflammatory bowel disease. Gastrointestinal endoscopy 2015: 81(3); 489-501.

2. Mowat $C$, Cole A, Windsor A, et al. Guidelines for the management of inflammatory bowel disease in adults. Gut 2011: 60; 571-607.

\section{PTH-087 REGIONAL IBD SURVEILLANCE ENDOSCOPY NORTH WEST (RISE NOW): A SURVEY OF CURRENT ENDOSCOPY PRACTITIONERS}

Gastroenterology Trainees Research and Improvement Network GasTRIN NoW*. Pennine Acute NHS Hospitals Trust, Greater Manchester, UK
Introduction Patients with longstanding ulcerative or Crohn's colitis are at an increased risk of developing colorectal cancer (CRC). Colitis related dysplasia appears to confer the greatest risk, prompting national societies to advocate colonoscopic surveillance. The SCENIC consensus recommends chromoendoscopy (CE) with high definition white light endoscopy (HDWLE) as the preferred technique followed by HDWLE without CE for dysplasia detection replacing traditional random biopsy sampling. ${ }^{1}$ Our aim was to explore practice, perceptions and barriers towards current 'gold standard' surveillance among endoscopists in North West England.

Method GasTRIN NoW investigators conducted a paper based survey of all colonoscopists in 13 hospitals in North West England to assess IBD colonoscopic practice between June and September 2018.

Results Forty two of 88 respondents (47.7\%) were consultant gastroenterologists. Seventy one respondents (81\%) performed IBD surveillance colonoscopy of which $5(7 \%)$ were surgeons. Of the endoscopists performing interval surveillance, 39\% $(n=28)$ had a specialist interest in IBD although 62\% $(n=40)$ felt that IBD surveillance endoscopy comprised less than a quarter of their endoscopic practice. Sixty six of the 71 colonoscopists (93\%) reported having access to CE but only $7 \%$ (4 of 60 responses) used $\mathrm{CE}$ in more than $50 \%$ of surveillance cases. Fifty seven percent of dye based CE users felt confident using this technique. Those with prior training in CE felt more confident performing CE than those without training (31 compared with $3, \mathrm{p}<0.05)$. Despite this, many colonoscopists still perform random biopsies (Figure 1). Among the 66 endoscopists with access to CE, 40 (61\%) and $27(42 \%)$ reported financial constraints and inadequate bowel preparation as barriers to CE surveillance respectively. Fifty nine percent of those performing surveillance endoscopy felt that their institution was open to training in new surveillance modalities.

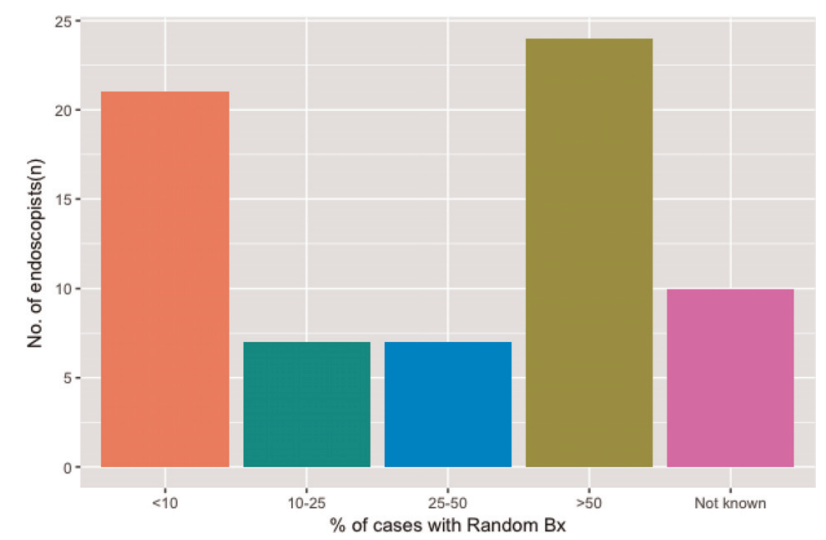

Abstract PTH-087 Figure 1 Percentage of cases with random biopsies

Conclusion IBD surveillance is predominantly delivered by consultant gastroenterologists with $\mathrm{CE}$ access being widely available. Despite this, WLE with random biopsy sampling is still widely practiced even by gastroenterologists. Lack of confidence from perceived lack of training, financial constraints and poor bowel preparation were cited as main reasons for not performing CE. Overcoming operational barriers may positively impact on quality of surveillance in IBD patients. 


\section{REFERENCES}

1. Laine L, Kaltenbach T, Barkun A, et al., SCENIC international consensus statement on surveillance and management of dysplasia in inflammatory bowel disease, Gastrointestinal endoscopy, 2015:81(3);489-501.

\section{PTH-088 QUALITY OF CARE IN IBD PATIENTS TRANSFERRING BETWEEN HEALTHCARE PROVIDERS}

Gastroenterology London Investigative Network for Trainees (GLINT) Gastroenterology London Investigative Network for Trainees GLINT** Gastroenterology London Investigative Network for Trainees, London, UK

\subsection{6/gutjnl-2019-BSGAbstracts. 147}

Introduction Continuity of care is vital in managing IBD. Many patients with IBD are young and more likely to change location frequently. However little is known about the quality and impact of a transfer of care between gastroenterologists. This study aims to assess the quality of information provided when patients are referred to a new provider and assess the impact on disease.

Methods The GLINT network retrospectively audited outpatient IBD referrals between 1st Jan and 28thFeb 2018. Patients with an existing diagnosis of IBD transferring to a new secondary or tertiary healthcare provider were included. Hospital records were assessed using Cornerstones Health 'IBD Checklist for Care Continuity ${ }^{\mathrm{TM}}$. A positive outcome was defined as the absence of a primary care attendance for IBD, clinically-diagnosed disease flare, steroid prescription or hospitalisation in the 6 months post referral. Discrete variables were analysed by Fisher's Exact test and continuous variables by Kruskall-Wallis (corrected for multiple comparisons).

Results 149 cases were identified from 16 hospitals with a median of 11 (IQR 4-15) patients per hospital. Diagnoses were CD in $44 \% \quad(n=66)$, UC in $54 \% \quad(n=81)$ and IBD unclassified in $1 \%(n=2)$. The median age of patients was 31 (IQR 25-45) years.

The sources of the referral letter were primary care (PC) $(\mathrm{n}=101,68 \%)$, secondary care (SC) $(\mathrm{n}=3624 \%)$ and private practice $(\mathrm{PP})(\mathrm{n}=11,7 \%)$. The reason for transfer included re-location $(n=75,50 \%)$, tertiary opinion $(n=35,23 \%)$, transferring from PP $(n=23,15 \%)$ and transfer from paediatric care $(\mathrm{n}=8,5 \%)$.

The referral letters received from SC included a significantly greater median number of data points (4, IQR 3-5, $\mathrm{n}=36)$ compared with from PC (2, IQR 2-3, $\mathrm{n}=101$, $\mathrm{p}<0.0001)$, but not significantly more than from PP (3, IQR $2-5, \mathrm{n}=11, \mathrm{p}=0.06$ ). Referrals from SC were more likely to include the most recent endoscopy report compared to those from PC $(51 \%$ vs $22 \%, \mathrm{p}=0.002)$ and the latest imaging (44\% vs $11 \%, \mathrm{p}<0.0001)$. Other data points including medication history were equally well provided by either source (86\% vs $89 \%$, n.s.).

Positive outcome was associated with the inclusion of more than 3 data points (OR 2.266, 95\% CI 1.092 - 4.569, $\mathrm{p}=0.03$ ), and specifically the inclusion of the most recent imaging (OR 2.844, 95\% CI 1.185 - 7.19, p=0.04). Referrals from SC were associated with a positive outcome compared to those from PC (OR 2.941, 95\% CI 1.264 - 7.021, $\mathrm{p}=0.01$ ).

Conclusion This multicentre audit of IBD centres in London demonstrates that referrals pertaining to a transfer of care often lack key pieces of clinical data. Most referrals come from PC, yet tend to include less information, possibly due to a lack of access to investigation results in PC. Hence, the IBD transfer of care checklist and greater involvement of SC in this process may improve the quality of information provided and ultimately positively impact on outcome.

\section{PTH-089 RATES OF WOUND HEALING IN PATIENTS WITH CROHN'S DISEASE UNDERGOING PROCTECTOMY}

${ }^{1}$ Rebecca Grant*, ${ }^{2}$ Sonia Bouri, ${ }^{2,3}$ Alfonso Elosua-González, ${ }^{2}$ Stella Dilke, ${ }^{2}$ Kapil Sahnan, ${ }^{2}$ Samuel Adegbola, ${ }^{2}$ Janindra Warusavitarne, ${ }^{2}$ Philip Tozer, ${ }^{2}$ Ailsa Hart. ${ }^{1}$ Royal Infirmary of Edinburgh, Edinburgh, UK; ${ }^{2}$ St Mark's Hospital, Harrow, UK; ${ }^{3}$ Complejo Hospitalario de Navarra, Navarra, Spain

\subsection{6/gutjnl-2019-BSGAbstracts. 148}

Introduction About 20\% patients with perianal Crohn's (pCD) undergo proctectomy with a significant number developing unhealed wounds. The purpose of this study was to determine factors which may be associated with poor wound healing in patients with $\mathrm{pCD}$ who had undergone proctectomy in the biologics era.

Methods Case record review was carried out of 79 patients with pCD who underwent proctectomy at St Mark's Hospital between 2005 and 2017. Healing rates at 6 and 12 months post proctectomy were considered and univariate regression analysis was performed.

Results Complete data regarding healing were available for $97.5 \%(77 / 79)$ at 6 months and $100 \%$ at 12 months. $45 / 77$ (43.7\%) patients had failure of wound healing at 6 months and $34 / 79(33 \%)$ at 12 months. A younger age at diagnosis of Crohn's disease was significantly associated with failure of healing at 12 months (median age $21 \pm 9.7$ unhealed; median age $27 \pm 13.6$ healed; $p=0.03)$.

$76.7 \%(61 / 79)$ patients received biologic treatments prior to proctectomy, however exposure to biologics was not a significant factor in predicting failure of wound healing (Infliximab $\mathrm{p}=0.74$; Adalimumab $\mathrm{p}=0.57$; Vedolizumab $\mathrm{p}=0.21$ ). Current smoking status was not associated with poor wound healing $(\mathrm{p}=0.18)$.

Other parameters which were not associated with failure of wound healing in our cohort included gender, corticosteroid exposure in the previous one month, thiopurine exposure in previous 3 months, number of biologics exposed to, perianal sepsis on MRI within the last 12 months, Montreal Classification, duration of CD prior to proctectomy, albumin and CRP. Conclusion A third of patients have unhealed wounds after 1 year follow-up after protectomy. A younger age at diagnosis of Crohn's disease was the only factor associated with an unhealed perineal wound; this may in part be due to more severe disease progression in patients diagnosed at a younger age. Larger scale studies are required to determine if other parameters such as exposure to biologics play a role in predicting rates of wound healing.

\section{PTH-090 A NEW MODEL OF CONSULTATION TO FACILITATE A PATIENT CENTRED APPROACH IN THE IBD CLINIC}

Suzanne Tattersall, Shahd Ahmed, Francesca Fairhurst, Veronica Hall*, George Lipscomb, Zandile Ndlovu, Salil Singh. Royal Bolton Hospital, Bolton, UK

\subsection{6/gutjnl-2019-BSGAbstracts.149}

Introduction The consultation is a pivotal event in the clinician/patient relationship, defined as the act of seeking 